\title{
Total Syntheses Supramolecular Style: Solid-State Construction of [2.2]Cyclophanes with Modular Control of Stereochemistry
}

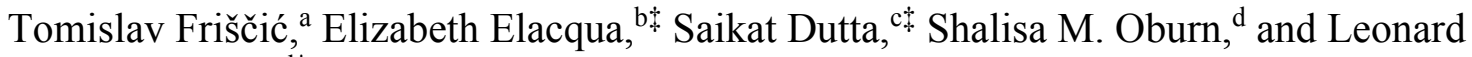
R. MacGillivray ${ }^{\mathrm{d}^{*}}$

a Department of Chemistry, McGill University, 801 Sherbrooke Street West H3A0B8 Montreal, Canada

${ }^{\mathrm{b}}$ Department of Chemistry and Materials Research Institute, Pennsylvania State University, University Park, PA 16802.

${ }^{\mathrm{c}}$ Department of Chemistry, National Institute of Technology Karnataka, Surathkal Mangalore, Karnataka, India

d Department of Chemistry, University of Iowa, Iowa City, IA, 52245-1297, USA

Author email address: len-macgillivray@uiowa.edu

\section{SUPPLEMENTARY INFORMATION}

1) Syntheses

2) X-ray diffraction

3) ${ }^{1}$ H NMR spectroscopy

4) Optical spectroscopy

5) References 


\section{1) Syntheses}

Chemicals. All chemicals were purchased from Sigma Aldrich and used without further purification.

meta-di-[2-(4-pyridyl)ethenyl] (m-bpeb). The diolefin m-bpeb was prepared using a twophase Pd-coupling reaction from 1,3-dibromobenzene and 4-vinylpyridine. In a typical experiment, 1,3-dibromobenzene $(2.84 \mathrm{~g}, 10 \mathrm{mmol})$ was mixed with 4-vinylpyridine $(3.34 \mathrm{~g}$, $30 \mathrm{mmol}, 1.5$ equiv.) and a palladium (II) chloride catalyst (20 $\mathrm{mg}, 0.17 \mathrm{mmol}$ ), followed by of tri(o-tolyl)phosphine $(150 \mathrm{mg}, 0.49 \mathrm{mmol})$. After stirring at room temperature for several minutes, aq. potassium carbonate solution $\left(50 \mathrm{~mL}, c=0.38 \mathrm{~mol} \mathrm{dm}^{-3}\right)$ was added, followed by of tri(n-butyl)amine $(0.5 \mathrm{~mL})$ as the phase transfer reagent. The suspension was refluxed for $17 \mathrm{~h}$. The reaction mixture was allowed to cool to room temperature and the resulting white precipitate was extracted with chloroform $(5 \times 50 \mathrm{~mL})$ and dried over anhy. $\mathrm{Na}_{2} \mathrm{SO}_{4}$. Solvent was evaporated under vacuum to yield $\mathbf{m}$-bpeb ${ }^{1}$ as a white powder $(2.1 \mathrm{~g}, 75 \%)$.

ortho-di-[2-(4-pyridyl)ethenyl] (o-bpeb). The diolefin reactant o-bpeb was prepared using a double Wittig condensation reaction involving 1,2-benzenebis(triphenylphosphonium) bromide $^{2}$ and pyridine-carboxaldehyde. In a typical experiment 1,2benzenebis(triphenylphosphonium) bromide $(8.5 \mathrm{~g}, 11 \mathrm{mmol})$ was mixed with pyridine-4carboxaldehyde $(2.70 \mathrm{~g}, 25 \mathrm{mmol}, 1.10$ equiv. $)$ in ethanol $(30 \mathrm{~mL})$. An ethanolic solution of lithium ethoxide $\left(100 \mathrm{~mL}, \mathrm{ca} .=0.4 \mathrm{~mol} \mathrm{dm}^{-3}\right)$ was added to the reaction mixture. The resulting orange reaction mixture was stirred for $30 \mathrm{~min}$. at room temperature and subsequently refluxed for $2 \mathrm{~h}$. The solvent was removed under vacuum and a saturated solution of $\mathrm{HCl}$ in ethanol was added to the remaining oily residue. The resulting yellow precipitate was filtered, dried for 24 h. at $60{ }^{\circ} \mathrm{C}$, and then was stirred in an solution of $10 \% \mathrm{NaOH}(30 \mathrm{~mL})$ for 24 hours. The resulting white precipitate was filtered then washed with water and dried at $60{ }^{\circ} \mathrm{C}$ to yield $\mathbf{0}$ bpeb $^{3}(0.74 \mathrm{~g}, 26 \%)$.

Representative synthesis of co-crystals. Co-crystals of 2(4-Cl-res) 2 (m-bpeb) were obtained via slow solvent evaporation. Specifically, m-bpeb $(200 \mathrm{mg}, 0.70 \mathrm{mmol})$ and 4Cl res $(112 \mathrm{mg}, 0.77 \mathrm{mmol}, 1.1 \mathrm{~mol}$. eq.) were dissolved in nitromethane $(15 \mathrm{~mL})$. The solution was heated to boiling and cooled slowly to ambient temperature. The co-crystals were isolated by vacuum filtration, washed with a small amount of nitromethane and air dried. Co-crystals of 2(4-Cy res) 2 (m-bpeb) and 2(4-Cl res) 2 (o-bpeb) were obtained in the same manner described above.

2(4-Cl res) 2 (o-bpeb) ${ }^{1} \mathrm{H}$ NMR (400 MHz, DMSO-d $) \delta: 9.86(\mathrm{~s}, 1 \mathrm{H}), 9.39(\mathrm{~s}, 1 \mathrm{H}), 8.53$ (dd, $J=1.6,4.6 \mathrm{~Hz}, 4 \mathrm{H}), 7.97(\mathrm{~d}, J=16.2 \mathrm{~Hz}, 2 \mathrm{H}), 7.74(\mathrm{~m}, 2 \mathrm{H}), 7.63(\mathrm{~d}, J=4.8 \mathrm{~Hz}$, 4H), $7.15(\mathrm{~m}, 2 \mathrm{H}), 7.12(\mathrm{~d}, J=16.2 \mathrm{~Hz}, 2 \mathrm{H}), 7.00(\mathrm{~d}, J=8.7 \mathrm{~Hz}, 1 \mathrm{H}), 6.38(\mathrm{~d}, J=2.7$ $\mathrm{Hz}, 1 \mathrm{H}), 6.17(\mathrm{dd}, J=2.8,8.6 \mathrm{~Hz}, 1 \mathrm{H})$.

2(4-Cl res) 2 (m-bpeb) ${ }^{1} \mathrm{H}$ NMR (400 MHz, DMSO-d $)$ ) $\delta: 9.94$ (s, 1H), 9.46 (s, 1H), 8.57 $(\mathrm{dd}, J=2.0,6.1 \mathrm{~Hz}, 4 \mathrm{H}), 7.98(\mathrm{~s}, 1 \mathrm{H}), 7.61-7.57(\mathrm{~m}, 8 \mathrm{H}), 7.44(\mathrm{t}, 1 \mathrm{H}), 7.38(\mathrm{~d}, 2 \mathrm{H}), 7.05$ $(\mathrm{d}, J=11.5 \mathrm{~Hz}, 1 \mathrm{H}), 6.42(\mathrm{~d}, J=3.6 \mathrm{~Hz}, 1 \mathrm{H}), 6.20(\mathrm{dd}, J=3.6,11.2 \mathrm{~Hz}, 1 \mathrm{H})$. 
2(4-Cy res)·2(m-bpeb) ${ }^{1} \mathrm{H}$ NMR (400 MHz, DMSO-d $\left.d_{6}\right) \delta$ : 9.02 (s, $\left.1 \mathrm{H}\right), 8.93$ (s, 1H), 8.57 (d, $J=7.9 \mathrm{~Hz}, 4 \mathrm{H}), 7.98(\mathrm{~s}, 1 \mathrm{H}), 7.62-7.57(\mathrm{~m}, 8 \mathrm{H}), 7.44(\mathrm{t}, 1 \mathrm{H}), 7.32(\mathrm{~d}, 2 \mathrm{H}), 6.80(\mathrm{~d}, J$ $=11.0 \mathrm{~Hz}, 1 \mathrm{H}), 6.23(\mathrm{~d}, J=3.2 \mathrm{~Hz}, 1 \mathrm{H}), 6.12(\mathrm{~d}, J=10.9 \mathrm{~Hz}, 1 \mathrm{H})$.

Photoreactions. Photoreactions were conducted using UV radiation from a $450 \mathrm{~W}$ medium-pressure mercury lamp in an ACE Glass photochemistry cabinet. In a typical experiment, co-crystals were finely ground using a mortar and pestle and placed between a pair of pyrex glass plates. The samples were irradiated in $10 \mathrm{~h}$ increments and mixed between consecutive irradiations. Product formation was monitored using ${ }^{1} \mathrm{H}$ NMR spectroscopy. Upon completion of the photoreaction, the products were isolated via base extraction with $\mathrm{CHCl}_{3}{ }^{4}$ 


\section{2) $X$-ray diffraction}

Single crystal X-ray diffraction experiments were performed on a Nonius Kappa CCD or a Bruker Neditonius APEX II Kappa diffractometer using Mo $K_{\alpha}$ radiation $(\lambda=$ $0.7107 \AA$ ). The structures were solved using direct methods and refined by full-matrix least-squares based on F2. All non-hydrogen atoms were refined using the anisotropic model. Hydrogen atoms bonded to carbon atoms were placed in idealized positions defined by the hybridization of the belonging carbon atom. The positions of hydrogen atoms bonded to oxygen atoms were calculated so as to establish the best possible hydrogen bonds to a nearest neighbor hydrogen bond acceptor. Structure solution and refinement were accomplished using ShelXT ${ }^{5}$ and ShelXL ${ }^{6}$, respectively. The structure was solved using direct methods. All non-hydrogen atoms were identified from the difference Fourier map and refined anisotropically.

In the X-ray refinement of $(\mathbf{m C p}-\mathbf{1}) \cdot 4($ benzene $) \cdot\left(\mathbf{H}_{2} \mathbf{O}\right)$, three guest benzene molecules exhibited high thermal disorder and the final structure model was refined excluding solvent benzene molecules using SQUEEZE routine of PLATON ${ }^{6}$ and modeled as a single benzene molecule. SQUEEZE output indicated a solvent accessible void volume of $661 \AA^{3}$ and an electron count of 121 consistent with three benzene molecules within the void. 
Table 1. Crystallographic and refinement data for mbpeb co-crystals and $\mathbf{m C p}$ products.

\begin{tabular}{|c|c|c|c|c|}
\hline Compound & $\begin{array}{c}2(\mathrm{Cl}- \\
\text { res }) \cdot 2(\mathrm{mbpeb}) \\
\end{array}$ & $\begin{array}{c}2(\mathrm{Cy}- \\
\text { res) } 2(\text { mbpeb }) \\
\end{array}$ & $\begin{array}{c}\text { endo,exo- } \\
\mathrm{mCp}-2\end{array}$ & $\begin{array}{c}\text { exo,exo-mCp- } \\
1 \\
\end{array}$ \\
\hline CCDC code & 1916587 & 1916584 & 1916583 & 1916585 \\
\hline Formula & $\mathrm{C}_{52} \mathrm{H}_{42} \mathrm{Cl}_{2} \mathrm{~N}_{4} \mathrm{O}_{4}$ & $\mathrm{C}_{32} \mathrm{H}_{32} \mathrm{~N}_{2} \mathrm{O}_{2}$ & $\mathrm{C}_{42} \mathrm{H}_{37} \mathrm{~N}_{5} \mathrm{O}$ & $\mathrm{C}_{57} \mathrm{H}_{51} \mathrm{~N}_{4} \mathrm{O}$ \\
\hline Formula weight & 857.49 & 476.59 & 627.76 & 808.57 \\
\hline Temp. & 210.15 & 190.15 & 190.15 & 190.15 \\
\hline Space group & P-1 & P-1 & $\mathrm{P} 2{ }_{1} / c$ & $\mathrm{C} 2 / \mathrm{c}$ \\
\hline $\mathbf{a}, \AA$ & $11.5691(13)$ & $9.5176(10)$ & 20.191(2) & $20.358(2)$ \\
\hline $\mathbf{b}, \AA$ & $15.0211(16)$ & $12.0260(12)$ & $15.4591(15)$ & $23.421(2)$ \\
\hline c, $\AA$ & $25.447(3)$ & $23.442(2)$ & $10.6486(11)$ & $11.3140(11)$ \\
\hline$\alpha$, deg. & $98.564(5)$ & $98.907(5)$ & 90 & 90 \\
\hline$\beta$, deg. & $96.316(5)$ & $100.093(5)$ & $90.677(5)$ & $122.402(5)$ \\
\hline$\gamma$, deg. & $91.647(5)$ & $97.630(5)$ & 90 & 90 \\
\hline volume, $\AA^{3}$ & $4341.8(8)$ & $2574.2(4)$ & $3323.6(6)$ & $4554.7(8)$ \\
\hline $\mathbf{Z}$ & 4 & 4 & 4 & 4 \\
\hline 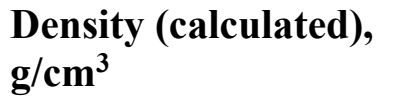 & 1.312 & 1.230 & 1.255 & 1.179 \\
\hline$\mu, \mathbf{m m}^{-1}$ & 0.202 & 0.076 & 0.077 & 0.070 \\
\hline $\begin{array}{l}2 \theta \text { range for data } \\
\text { collection, deg. }\end{array}$ & $4.384-45$ & $5.86-49.998$ & $\begin{array}{l}6.054- \\
50.064\end{array}$ & $5.576-49.924$ \\
\hline Reflections measured & 21974 & 16956 & 21986 & 14505 \\
\hline $\begin{array}{l}\text { Independent reflns. } \\
{[I>2 \sigma]}\end{array}$ & 11334 & 9046 & 5866 & 3998 \\
\hline $\begin{array}{l}\text { Data/restraints/para } \\
\text { meters }\end{array}$ & $11334 / 2232 / 1453$ & $9046 / 16 / 685$ & $5866 / 18 / 460$ & $3998 / 60 / 313$ \\
\hline $\mathbf{R}_{\text {int }}$ & 0.0801 & 0.0407 & 0.0352 & 0.0452 \\
\hline $\begin{array}{l}\text { Final R Indices } \\
{[I>2 \sigma]}\end{array}$ & $\begin{array}{l}R_{1}=0.1020 \\
w R 2=0.2720\end{array}$ & $\begin{array}{l}R_{1}=0.0467 \\
w R 2=0.0964\end{array}$ & $\begin{array}{l}R_{1}=0.0450 \\
w R 2= \\
0.1101\end{array}$ & $\begin{array}{l}R_{1}=0.0545, \\
w R 2=0.1406\end{array}$ \\
\hline R Indices (all data) & $\begin{array}{l}R_{1}=0.2240 \\
w R 2=0.3438\end{array}$ & $\begin{array}{l}R_{1}=0.0948 \\
w R 2=0.1138\end{array}$ & $\begin{array}{l}R_{1}=0.0572, \\
w R 2= \\
0.1185\end{array}$ & $\begin{array}{l}R_{1}=0.0868 \\
w R 2=0.1580\end{array}$ \\
\hline Goodness-of-fit on $\mathrm{F}^{2}$ & 1.018 & 1.009 & 1.045 & 1.033 \\
\hline
\end{tabular}


Table 2. Crystallographic and refinement data for obpeb co-crystals and $\mathbf{o C p}$ product.

\begin{tabular}{|c|c|c|}
\hline Compound & 2 (Cl-res) 2 (obpeb) & exo,exo-oCp \\
\hline CCDC code & 1916586 & 1916588 \\
\hline Formula & $\mathrm{C}_{52} \mathrm{H}_{42} \mathrm{Cl}_{2} \mathrm{~N}_{4} \mathrm{O}_{4}$ & $\mathrm{C}_{40} \mathrm{H}_{35} \mathrm{~N}_{4} \mathrm{O}_{1.5}$ \\
\hline Formula weight & 857.79 & 595.72 \\
\hline Temp. & 190.15 & 298.15 \\
\hline Space group & P-1 & P-1 \\
\hline $\mathbf{a}, \AA$ & $11.1645(11)$ & $8.5004(12)$ \\
\hline b, $\AA$ & $13.4581(13)$ & $11.8570(13)$ \\
\hline c, $\AA$ & $16.0572(16)$ & $16.676(3)$ \\
\hline$\alpha$, deg. & $113.861(5)$ & $91.326(7)$ \\
\hline$\beta$, deg. & $90.929(5)$ & $96.643(5)$ \\
\hline$\gamma$, deg. & $100.600(5)$ & $102.679(8)$ \\
\hline volume, $\AA^{3}$ & $2157.7(4)$ & $1626.7(4)$ \\
\hline $\mathbf{Z}$ & 2 & 2 \\
\hline Density (calculated), $\mathrm{g} / \mathrm{cm}^{3}$ & 1.320 & 1.216 \\
\hline$\mu, \mathbf{m m}^{-1}$ & 0.203 & 0.075 \\
\hline $2 \theta$ range for data collection, deg. & $6.16-50.148$ & $5.396-50$ \\
\hline Reflections measured & 14011 & 8696 \\
\hline Independent reflns. $[\mathrm{I}>\mathbf{2 \sigma}]$ & 7520 & 5616 \\
\hline Data/restraints/parameters & $7520 / 49 / 635$ & $5616 / 108 / 457$ \\
\hline $\mathbf{R}_{\text {int }}$ & 0.0362 & 0.0981 \\
\hline Final R Indices $[I>2 \sigma]$ & $\begin{array}{l}R_{1}=0.0460 \\
w R 2=0.0939\end{array}$ & $\begin{array}{l}R_{1}=0.0902 \\
w R 2=0.1557\end{array}$ \\
\hline R Indices (all data) & $\begin{array}{l}R_{1}=0.0952 \\
w R 2=0.1105\end{array}$ & $\begin{array}{l}R_{1}=0.3078 \\
w R 2=0.2314\end{array}$ \\
\hline Goodness-of-fit on $F^{2}$ & 1.006 & 0.959 \\
\hline
\end{tabular}




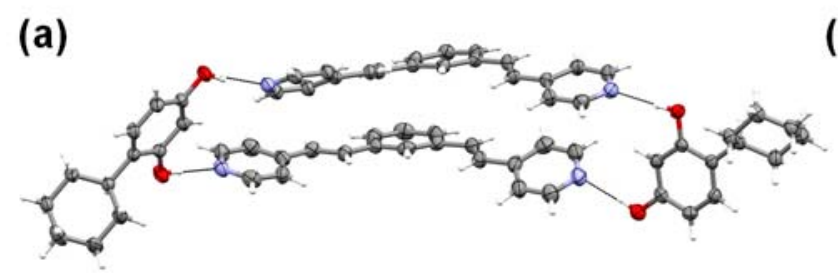

(b)

(c)

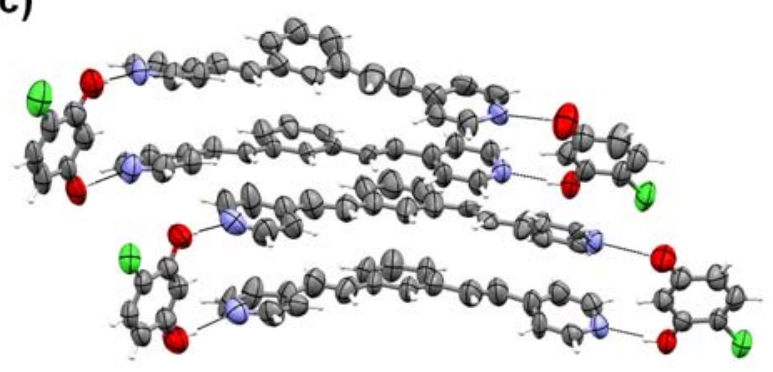

(d)

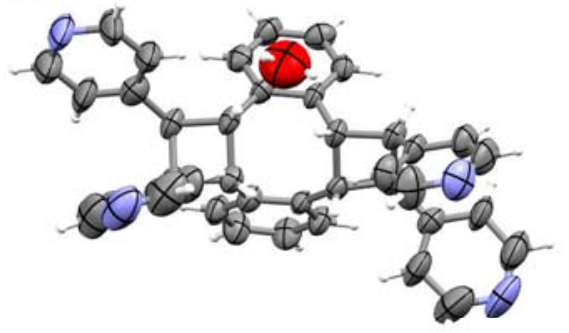

(e)

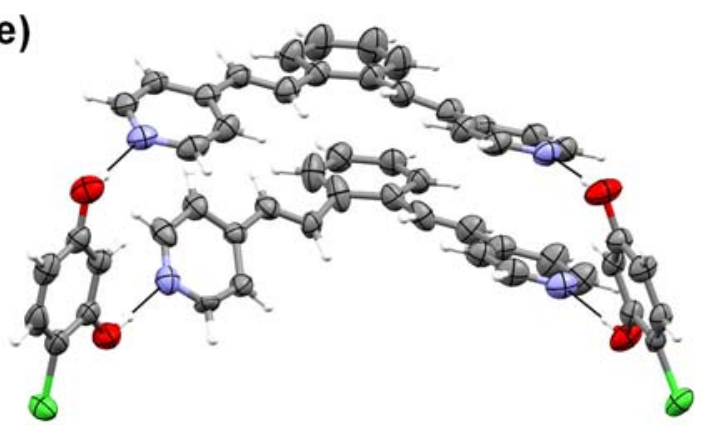

(f)
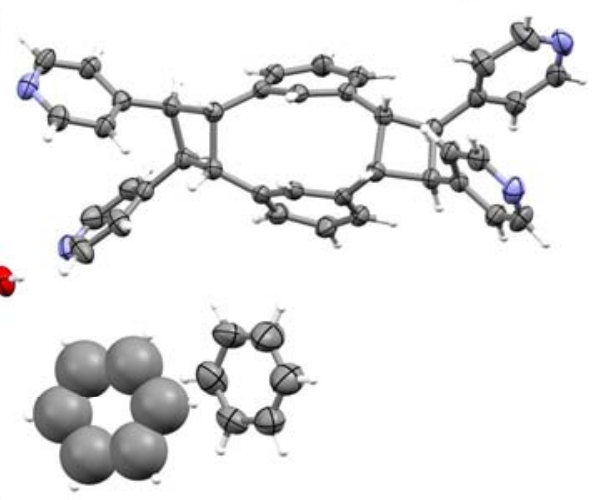

Figure S1. X-ray structures in ORTEP view of (a) 2(4-Cy-res) 2 (m-bpeb), (b) (oCp) $\cdot 2 \mathrm{H}_{2} \mathrm{O}$, (c) 2 (4-Cl-res) $\cdot 2\left(\right.$ m-bpeb), (d) (mCp-1) 4 (benzene) $\cdot \mathrm{H}_{2} \mathrm{O}$, (e) $2(4-\mathrm{Cl}-$ res) $2\left(\right.$ o-bpeb), and (f) $(\mathbf{m C p}-2) \cdot\left(\mathbf{C H}_{3} \mathbf{C N}\right) \cdot \mathrm{H}_{2} \mathrm{O}$. 

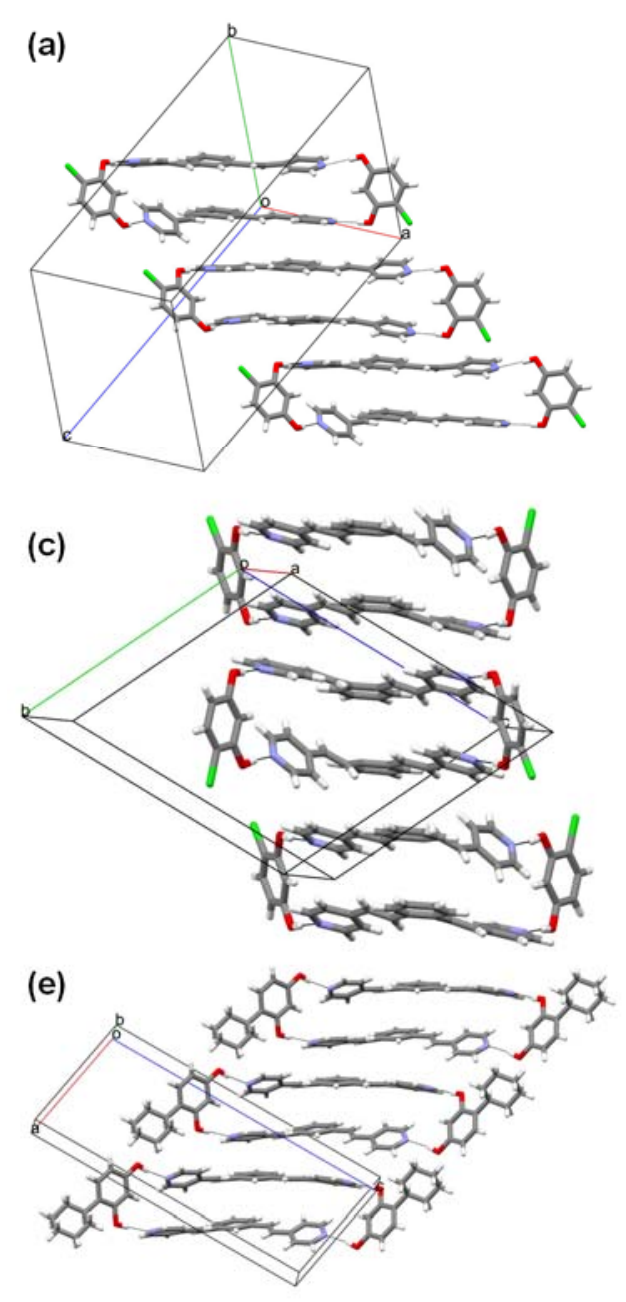

(b)
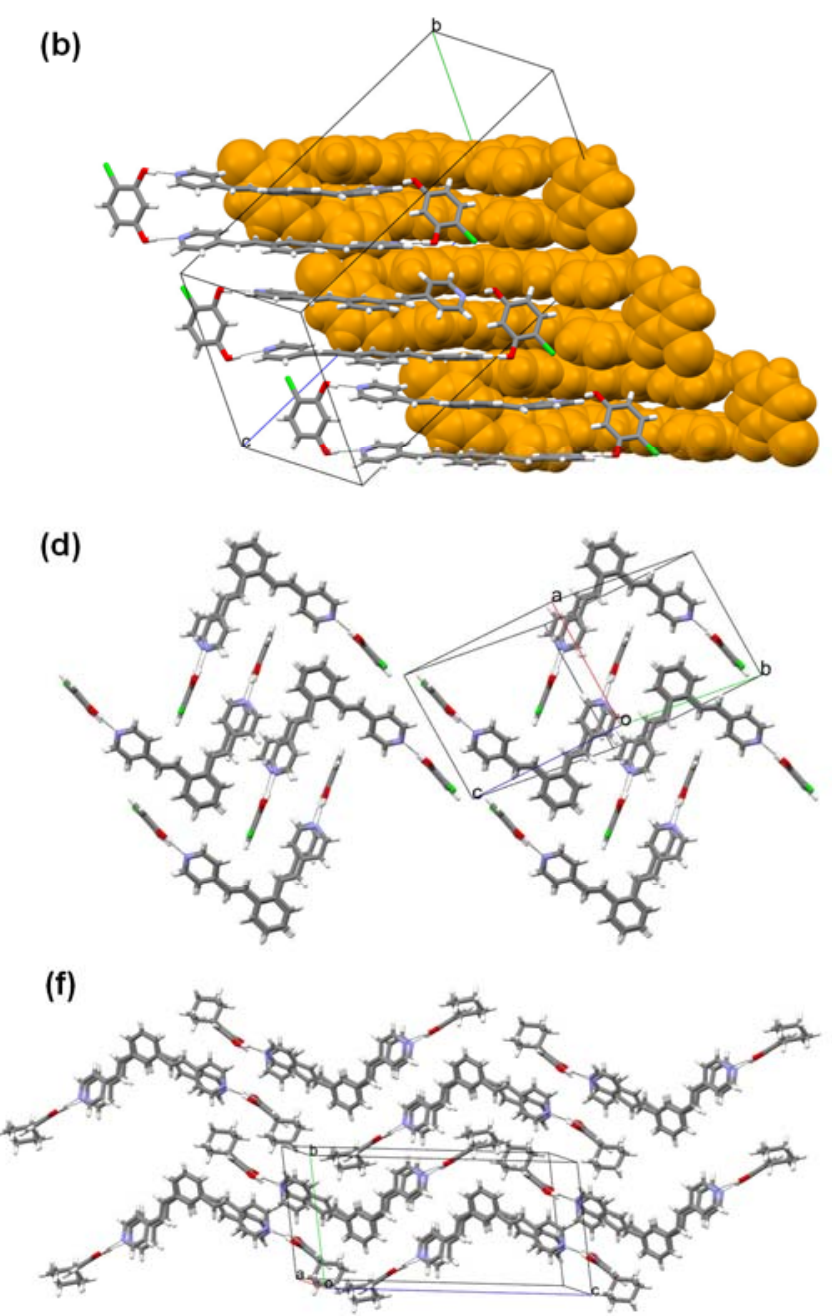

Figure S2. X-ray structures demonstrating packing of: (a) stacks of hydrogen-bonded 2(4Cl-res) 2 (m-bpeb), (b) extended packing of discrete assemblies of 2(4-Cl-res) 2 (m-bpeb) with molecules from figure (a) in spacefill view (orange), (c) stacks of hydrogen-bonded 2(4-Cl-res) 2(o-bpeb), (d) and extended packing of discrete assemblies of 2(4-Clres) 2 (o-bpeb) along the $c$-axis, (e) columns of 2(4-Cy-res) 2 (m-bpeb), (f) extended packing of discrete assemblies of $2(4-\mathbf{C y}-\mathbf{r e s}) \cdot 2(\mathbf{m}$-bpeb) along the $c$-axis. 
(a)

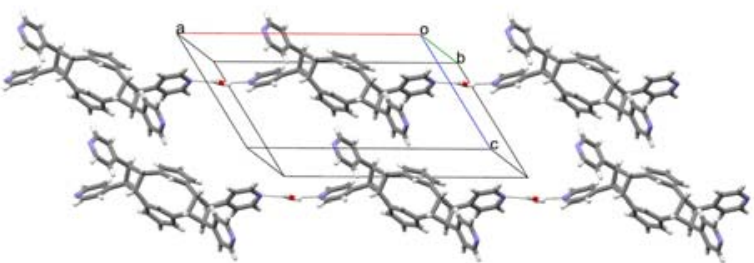

(b)

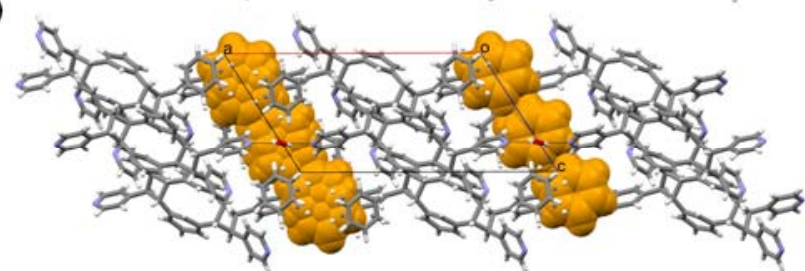

(c)

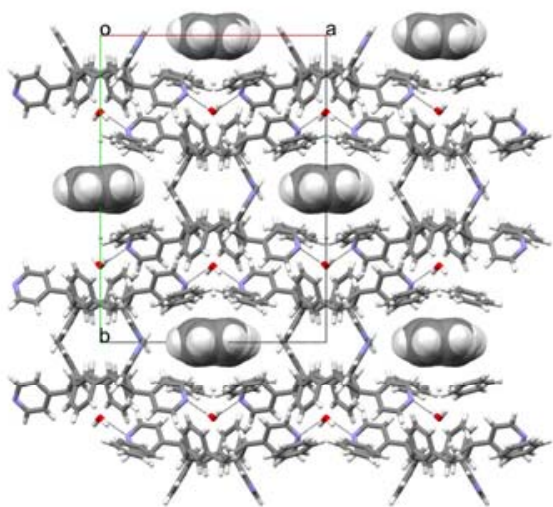

(d)

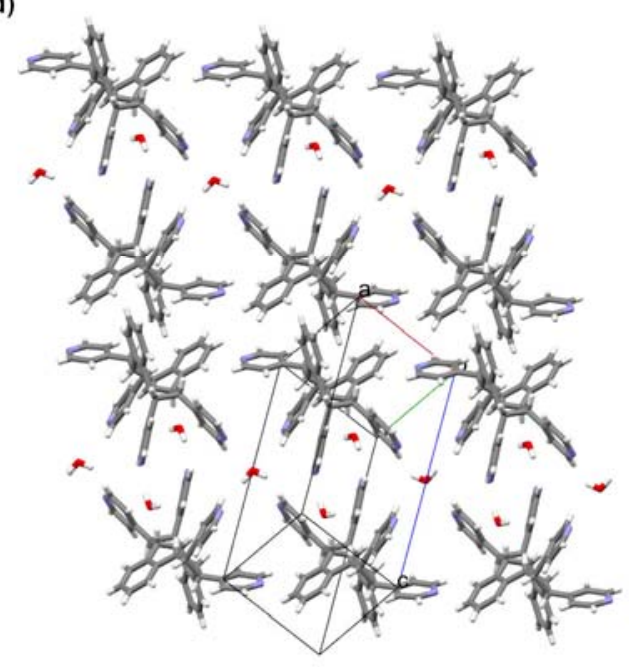

(e)

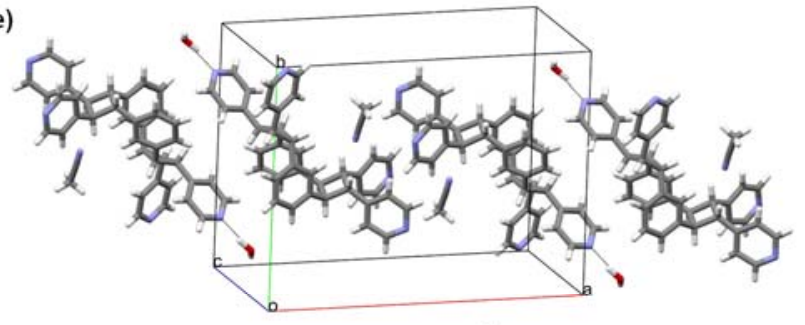

(f)

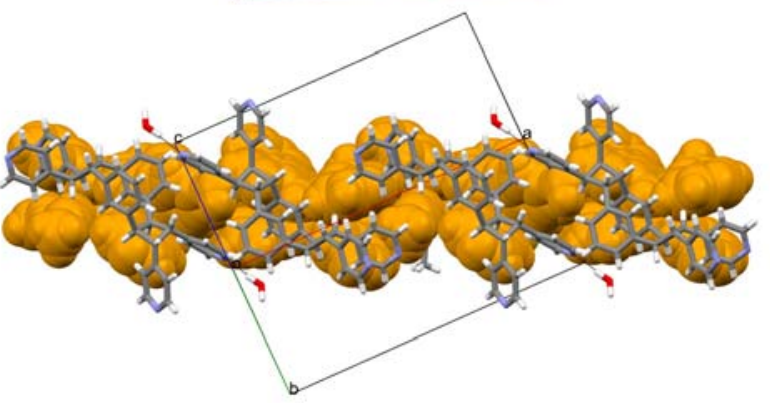

Figure S3. X-ray structures demonstrating packing of: (a) 1D hydrogen-bonded chains of (mCp-1) $\cdot 4$ (benzene) $\cdot \mathrm{H}_{2} \mathrm{O}$, (b) extended packing along the $c$-axis of $1 \mathrm{D}$ hydrogen bonded chains of (mCp-1) $\cdot 4$ (benzene) $\cdot \mathrm{H}_{2} \mathrm{O}$ with benzene represented in spacefill view (orange), (c) view of (mCp-1) $\cdot 4$ (benzene) $\cdot \mathrm{H}_{2} \mathrm{O}$ along the $c$-axis with channel occupied by benzene represented in spacefill view, (d) extended packing of $(\mathbf{o C p}) \cdot 2 \mathrm{H}_{2} \mathrm{O}$, (e) packing along the $a$-axis of $(\mathbf{m C p}-2) \cdot\left(\mathbf{C H}_{3} \mathbf{C N}\right) \cdot \mathrm{H}_{2} \mathrm{O}$, and (f) packing perpendicular to the $a$-axis of ( $\mathbf{m C p}$ 2) $\cdot\left(\mathbf{C H}_{3} \mathbf{C N}\right) \cdot \mathrm{H}_{2} \mathrm{O}$ with molecules from figure (e) in spacefill view (orange). 


\section{3) ${ }^{1} \mathrm{H}$ NMR spectroscopy}

The ${ }^{1} \mathrm{H}$ NMR spectra were recorded on Avance-400 operating at $400 \mathrm{MHz} .{ }^{1} \mathrm{H}$ chemical shifts were referenced from the chemical shifts of DMSO- $d_{6}$.

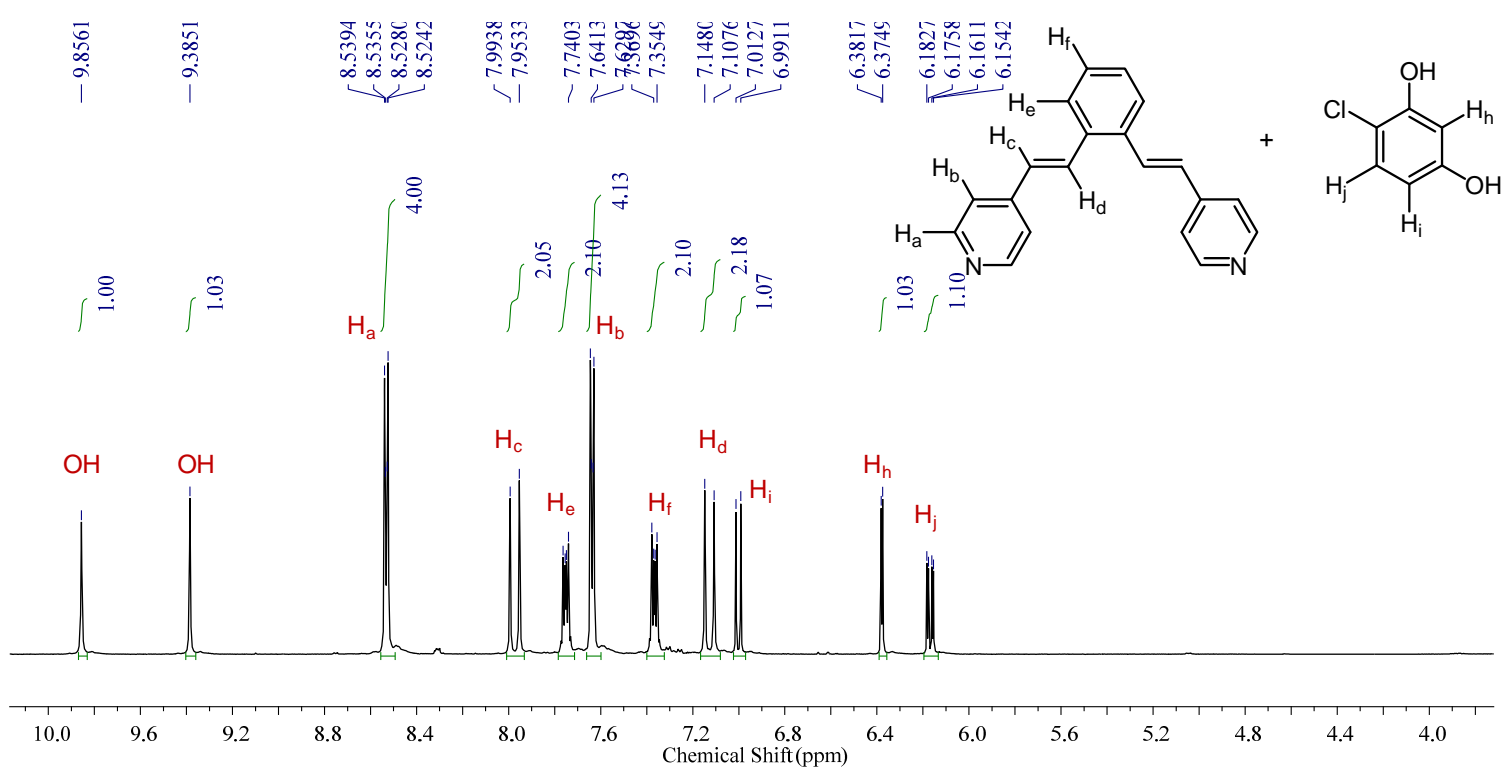

Figure S4a. ${ }^{1} \mathrm{H}$ NMR spectrum of 2(4-Cl-res) 2 (o-bpep) before photoreaction.

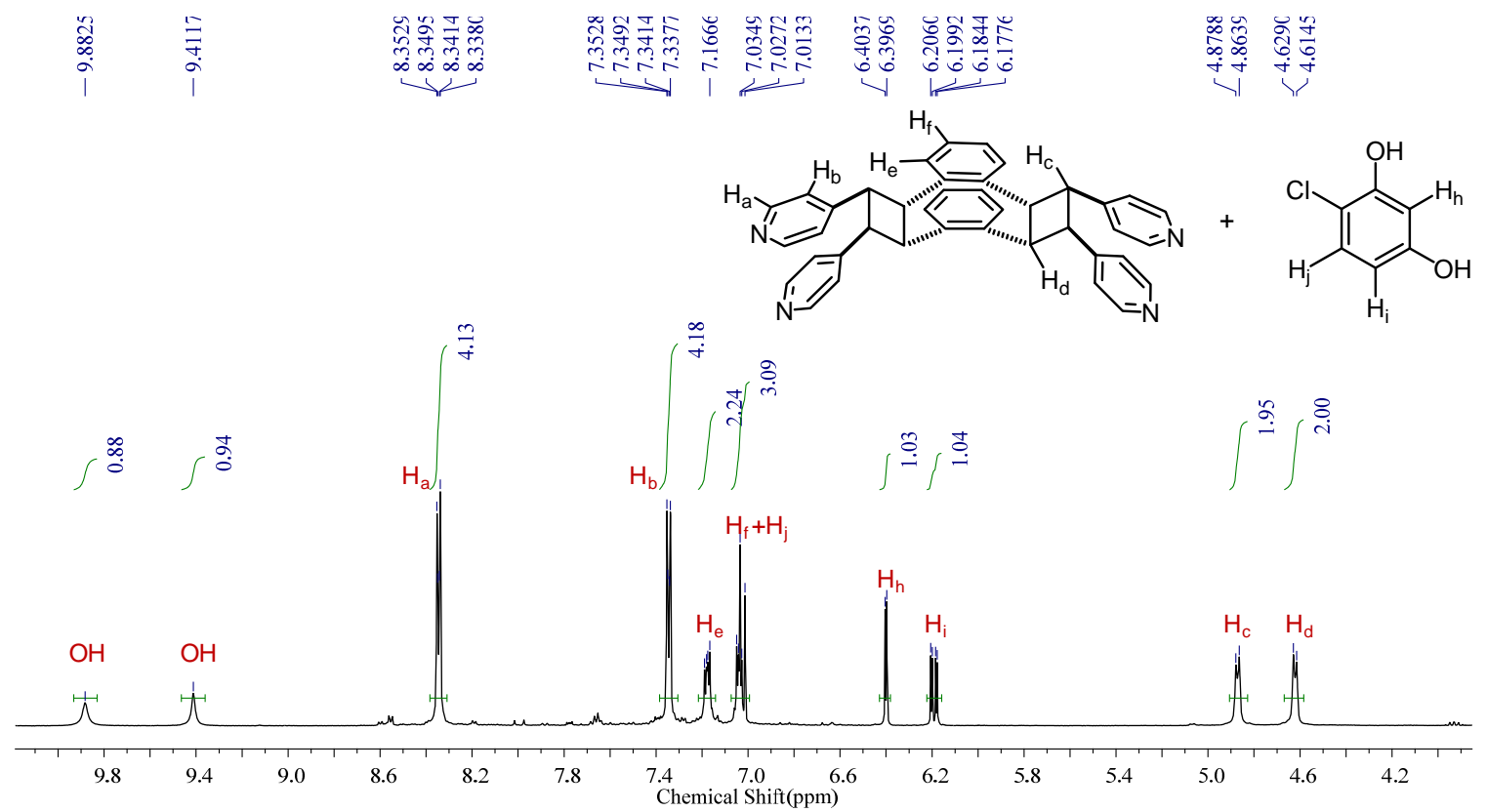

Figure S4b. ${ }^{1} \mathrm{H}$ NMR spectrum of 2(4-Cl-res) $2(\mathbf{o C p})$ after photoreaction. 


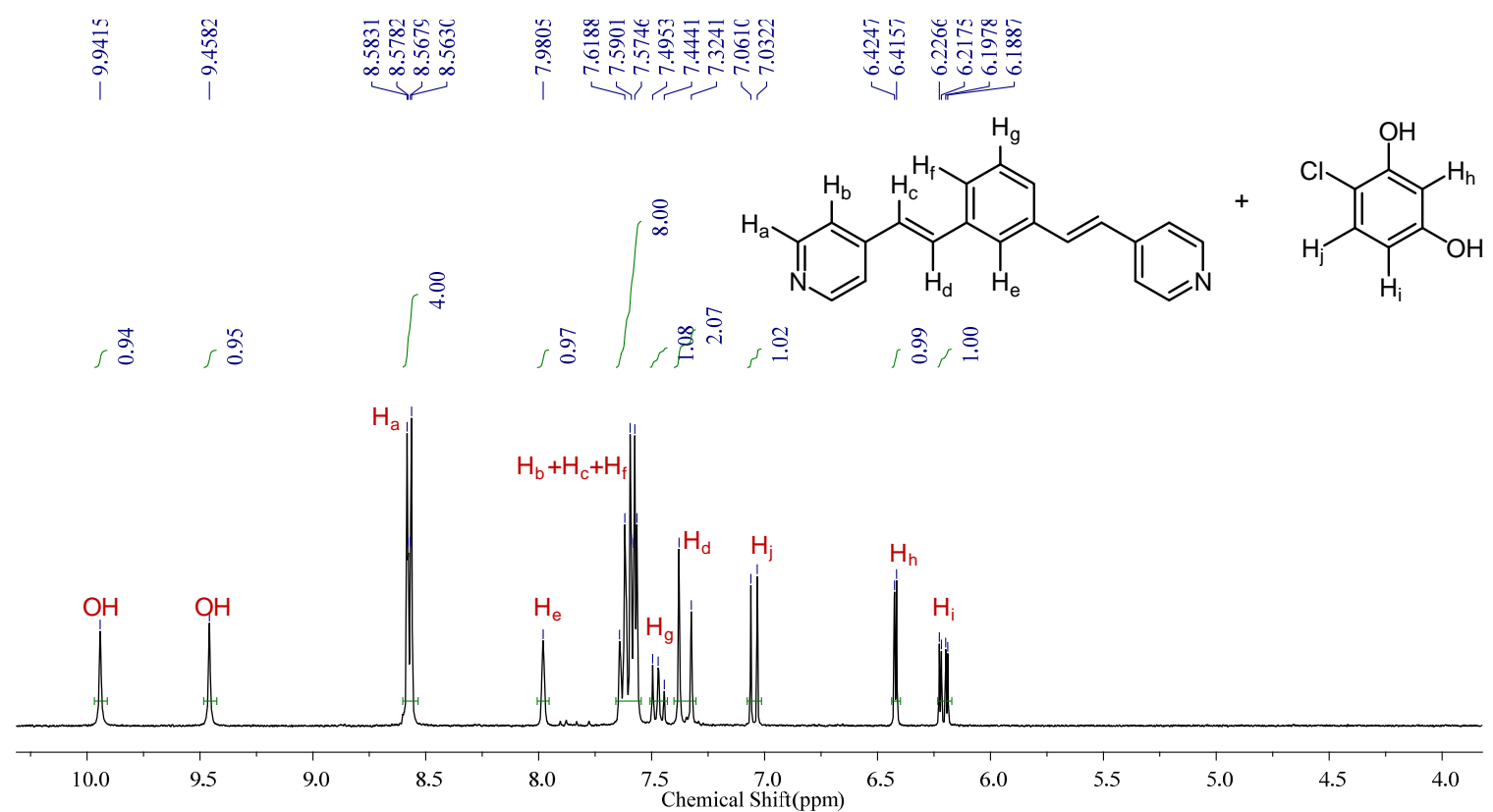

Figure S5a. ${ }^{1} \mathrm{H}$ NMR spectrum of 2(4-Cl-res) 2 (m-bpep) before reaction.

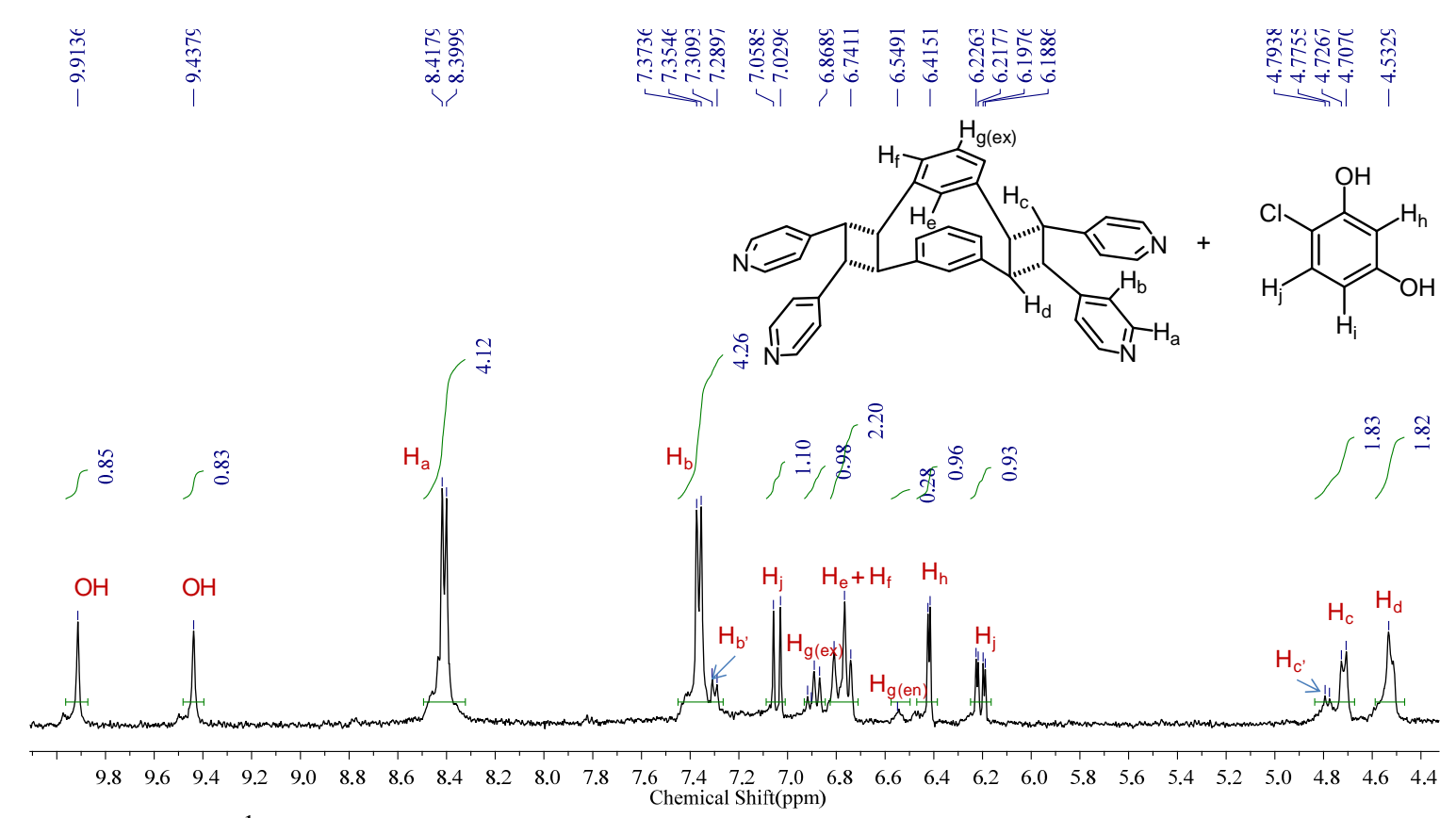

Figure S5b. ${ }^{1} \mathrm{H}$ NMR spectrum of 2(4-Cl-res) 2 (mCp-1)/ 2(4-Cl-res) 2 (mCp-2) after photoreaction. 


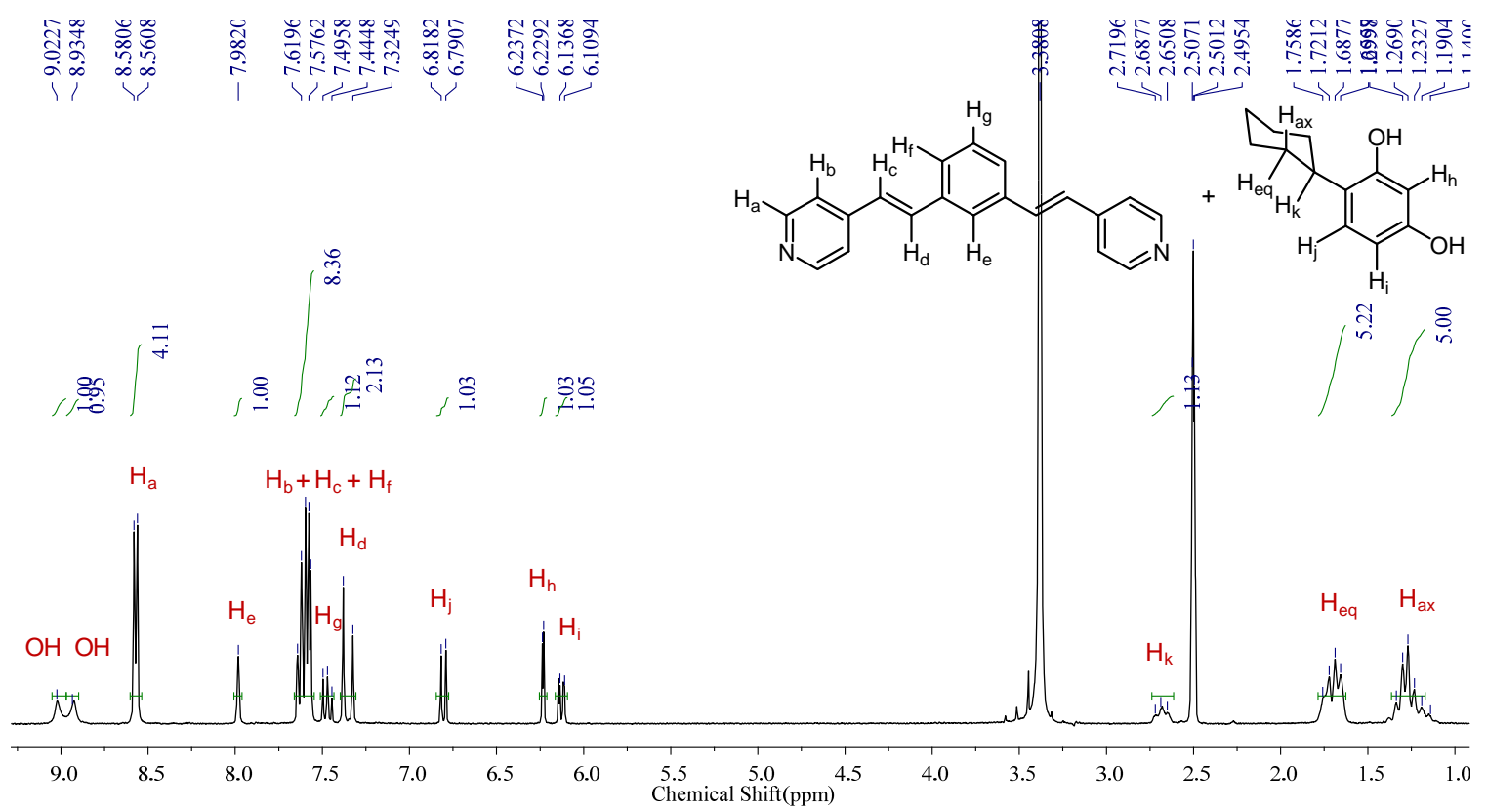

Figure S6a. ${ }^{1} \mathrm{H}$ NMR spectrum of 2(4-Cy-res) 2 (m-bpep) before reaction.

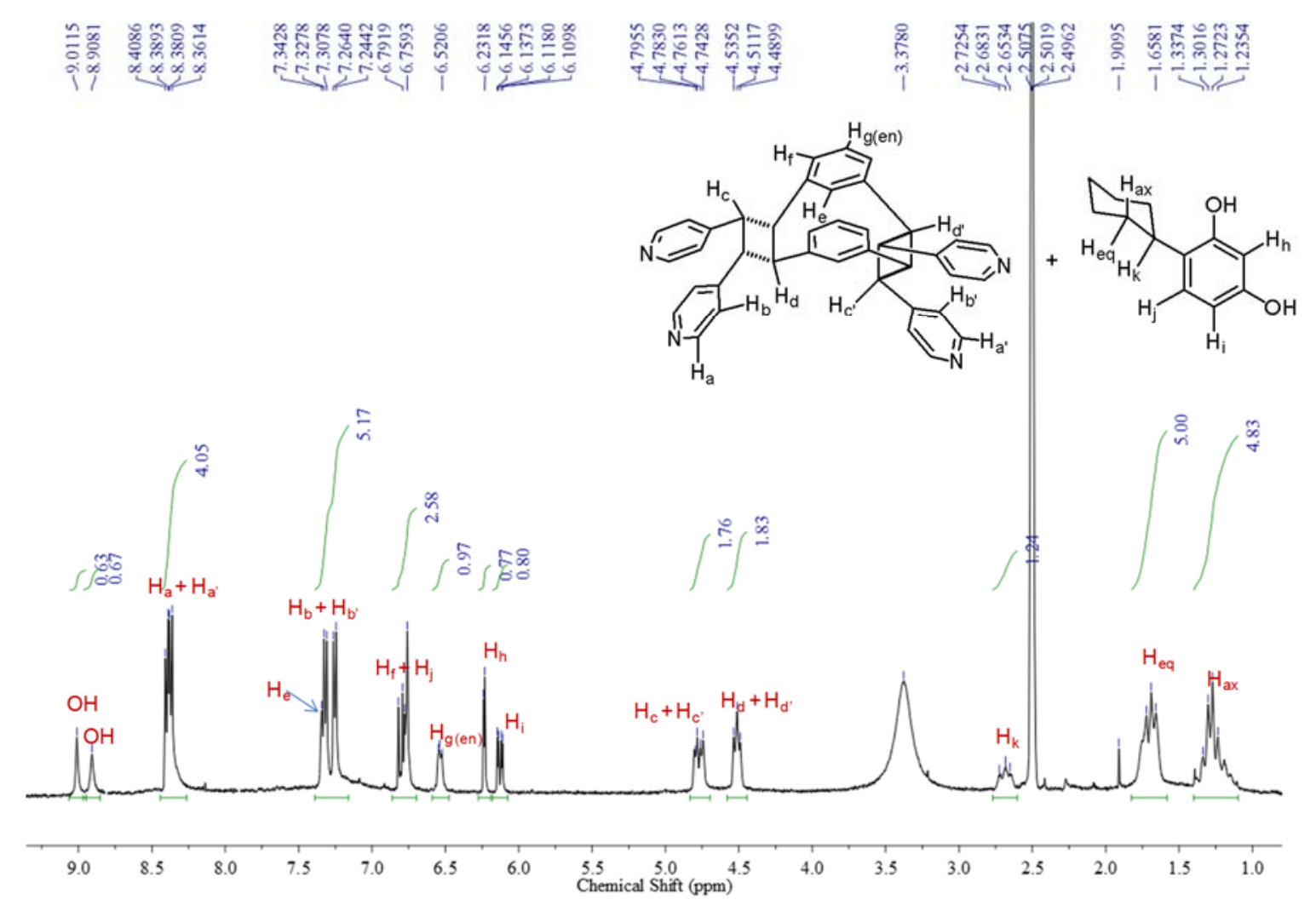

Figure S6b. ${ }^{1}$ H NMR spectrum of 2(4-Cy-res) $2(\mathbf{m C p}-\mathbf{1})$ after photoreaction. 


\section{4) Optical spectroscopy}

Absorption and emission measurements were obtained using a single channel AMINCO-Bowman Luminescence Spectrometer, Model FA-354 (Thermo Electron, Waltham, MA), with Xenon lamp excitation. Fluorescence Spectra were recorded in N,Ndimethylformamide at a scan rate of $5 \mathrm{~nm} / \mathrm{s}$ with both monochromators set to a $2 \mathrm{~nm}$ slit width.

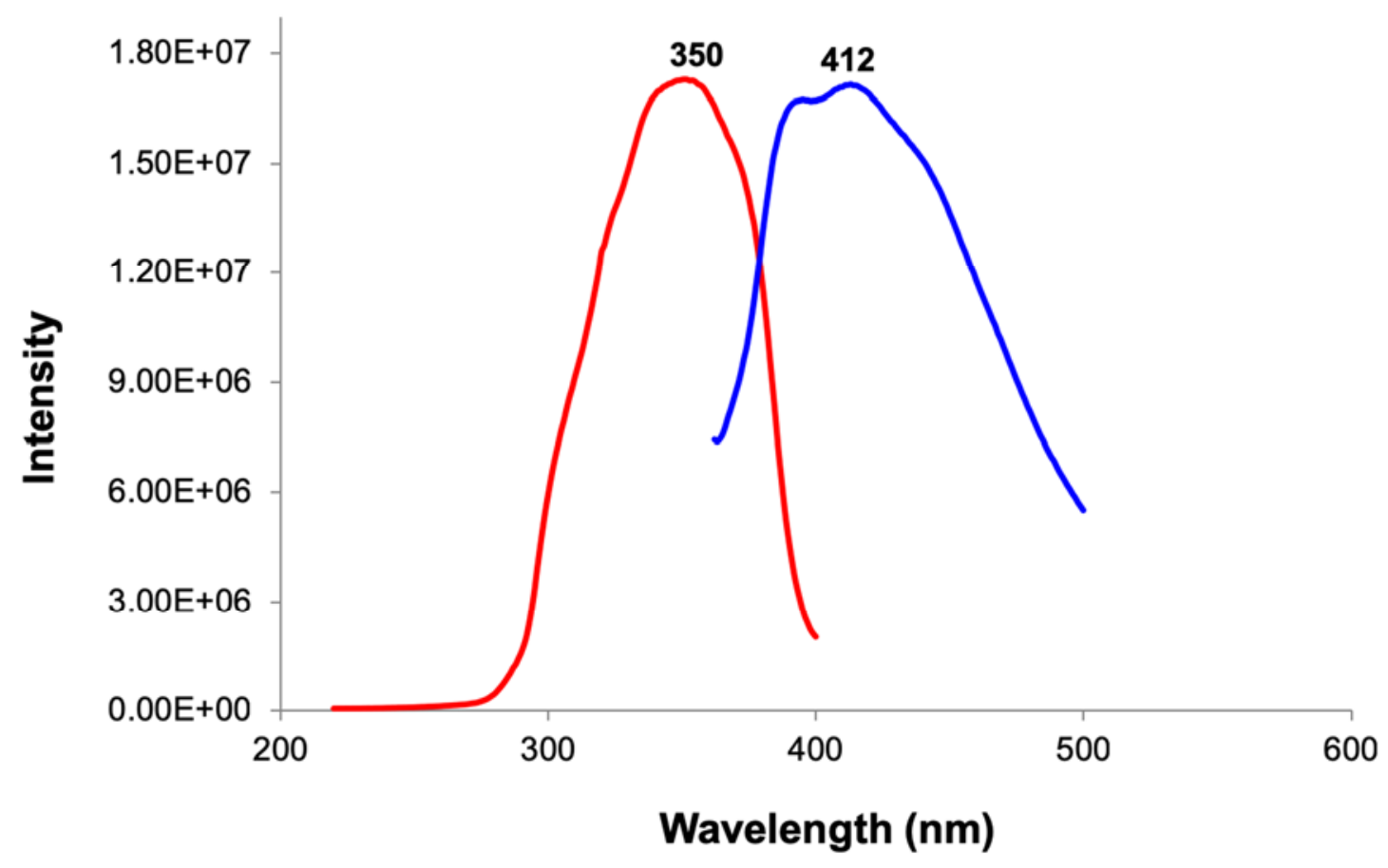

-EX with EM at 415 -EM with EX at 350

Figure S7a. Excitation (red) and emission (blue) spectra of mCp-1 and 2. 


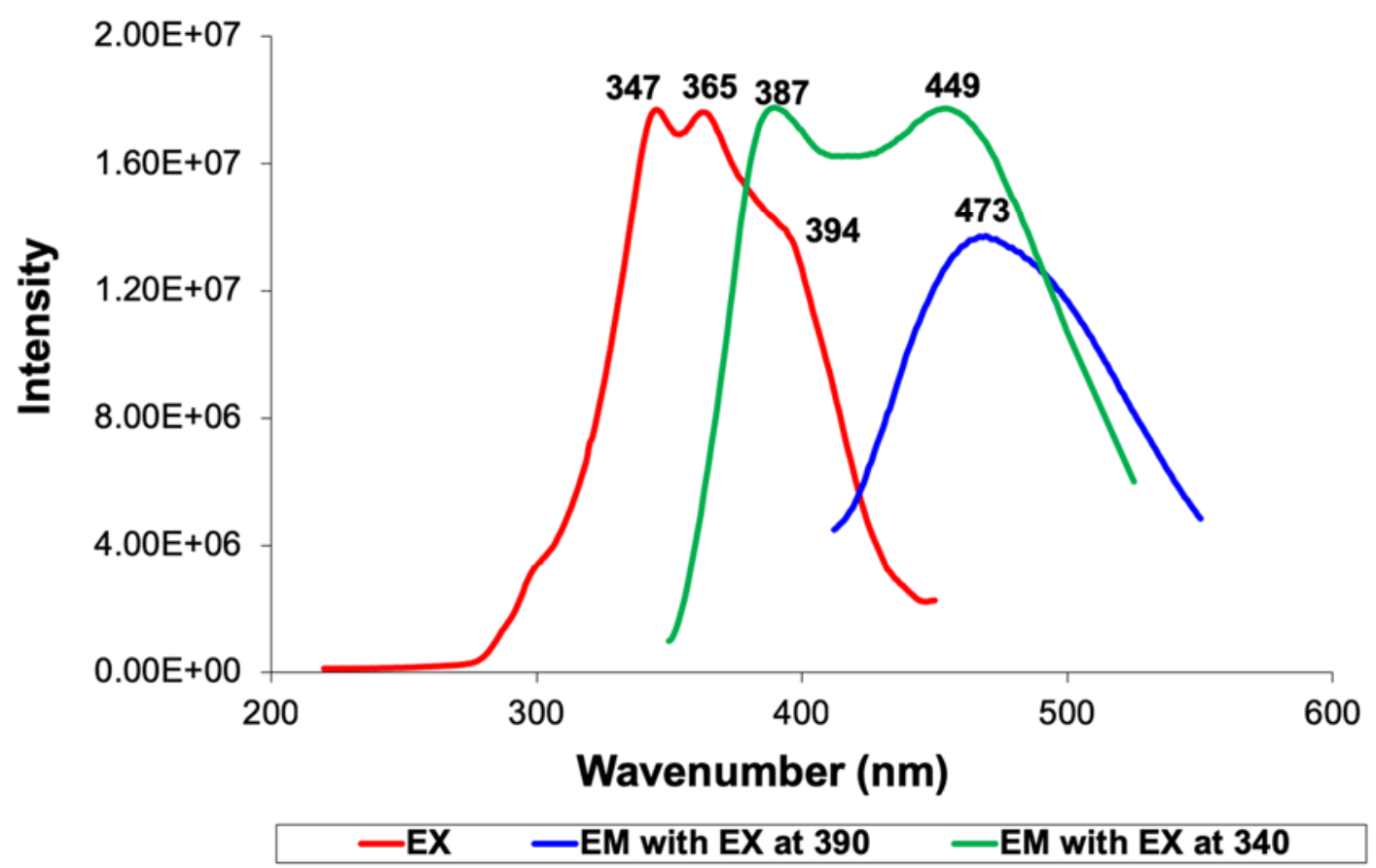

Figure S7b. Excitation (red) and emission (blue and green) spectra of oCp. 


\section{References:}

1. Bhowmik, P. K.; Nedeltchev, A. K.; Han, H., Tetrahedron Lett. 2007, 48, 5383.

2. Griffin, C. E.; Martin, K. R.; Douglas, B. E. J. Org. Chem. 1962, 27, 1627-1631.

3. S Šagud, I.; Milašinović, V.; Molčanov, K.; Marinić, Ž.; Škorić, I., J. Mol. Struct. 2018, 1171, 117.

4. MacGillivray, L. R.; Reid, J. L.; Ripmeester, J. A., J. Am. Chem. Soc. 2000, 122, 7817.

5. G. Sheldrick, Acta Crystallogr. Sect. A, 2015, 71, 3.

6. G. Sheldrick, Acta Crystallogr. Sect. C, 2015, 71, 3. 